\title{
Efficient Underwater Communication using Mobile Sink
}

\author{
Sushma $\mathbf{V}^{1}$ \\ Assistant Professor, Dept. of ISE, GSSSIETW, Mysuru, India ${ }^{1}$
}

\begin{abstract}
In Underwater Wireless Sensor Networks (UWSNs) energy, throughput are wide are of interest. These sensor networks make it possible in real time monitoring of the underwater environment. Major challenges that ar4e faced in such environment are low battery power, high bit error rates, low bandwidth availability etc. This paper mainly examines these challenges and possible solutions to overcome the problem by using a cluster formation among the nodes which communicate with a mobile sink. Our work mainly focuses the formation of cluster and path discovery of the cluster by the mobile sink. Finally, by using this strategy we prove that the energy used by sensor networks is lesser than other algorithms.
\end{abstract}

Keywords: Underwater Sensor Networks, Clustering, Energy Efficient, Mobile Sink.

\section{INTRODUCTION}

An underwater Underwater WirelessiSensor Networks (UWSNs) is a collection of organized and distributed wireless communication networks that comprises of enormous number of sensor nodes in underwater.[1] Although covering more than $70 \%$ of the Earth surface the oceans are not well known, due to its dimensions, difficulties of oceanographic data acquisition and the high costs of maritime operations. Nevertheless, there is an increasing interest on oceanographic data, due to its influence on the weather, fishing, navigation, biology, ecology and support for petroleum resources offshore exploration. The use of such sensors is increasing rapidly in many fields. These Underwater Sensor Networks are used for water quality surveillance, gas/oil spills monitoring, oceanographic data gathering, offshore survey or examination, submarine identification and diagnosis, catastrophe interception, pollution monitoring, military surveillance etc [2][3][4]. These underwater sensors resemble the terrestrial sensors. However, the UWSNs do not use radio signal or electromagnetic signals. Instead they make use of acoustic signals [5]. Due to the usage of acoustic communication, underwater sensors face large propagation delay, refraction, multipath interference, and high error rate and low communication bandwidth. There are many differentiations between underwater sensor networks and open ground environment.

The sensors in UWSNs are the relaying devices. They simultaneously gather and transmit data. It is very important that in a network all nodes achieve an equal share of bandwidth. Thus it can be easy to receive intended and entire information regarding the area under survey. Later on the beacon node sends the accumulated information towards the users, satellite or data center through radio or wired communication signals. There are many restrictions to the sensor nodes in underwater environment. Some of them are salinity, humidity, temperature, mobility of nodes, changing underwater environment.

Due to the high attenuation which are found or sensed in electromagnetic signals in water environment, it is not at all suitable for underwater communication [6].

A. Modulation Methods Used For Underwater Communication :

- Frequency Shift Keying : Earliest method of modulation used for underwater communication was FSK. It is the easiest form modulation which employs two different frequencies to modulate the data. Multi path reflection is one of the major challenges that the FSK faces in underwater environment. Thus this method.

- Phase Shift Keying : In this modulation, the phase of a reference signal is altered or changed in order to convey the sensed data. Finite number of phases is used to represent digital data where these phases are transformed into binary bits. After the modulation process in the modulator, the carrier signal passes or transmits the data to the receiver or demodulator. In demodulator, the changes in the phase of the received signals are identified. Thus the demodulator need not have any reference signal for this analysis purpose.[7]

- Frequency Hopped Spread Spectrum (FHSS) : FHSS is normally utilized in single-user FSK systems in order to provide channel clearing time in a multipath environment. In this modulation method the transmitter adopts various set 
of preordained frequency bins. These are processed progressively. Until the channels are idle reusability of the bins are avoided. This can be achieved by selecting the total number of frequency bins such that it exceeds the maximum multipath delay. This method is very simple to implement. But the drawback is that it has performance limitations in frequency-selective channels [8]

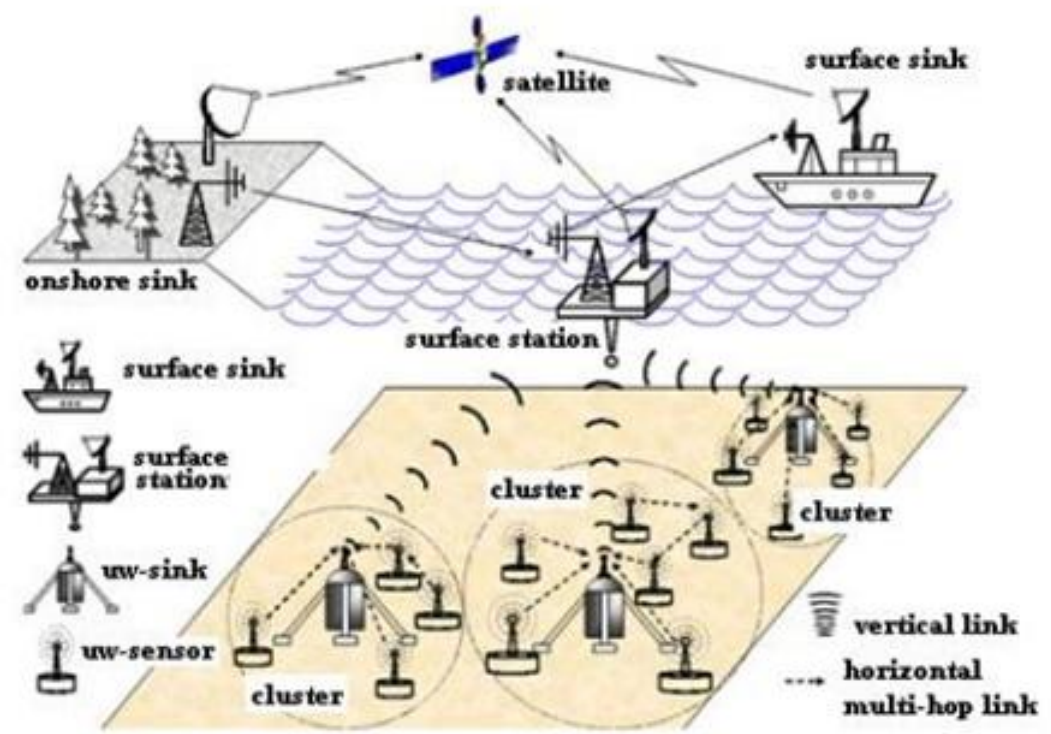

Fig. 1. A View Of UWSN Environment

B. Applications of Underwater Sensor Networks

- Seismic-Tracking - One of the advantageous application of underwater sensor are seismic-tracking/surveillance for withdrawal of oil/lubricant from subaqueous/marine areas [9]. "4 - D seismic" are widely utilized in determining the performance. It is also useful in the process of motivation intervention.

- Underwater cyborg - This supports groups of underwater autonomous cyborg which are also known as robots that can eventually sense, collect and transmit data such as chemical leakage or biological phantasm like phytoplankton flocking and also equipment managing [10].

C. Challenges of Underwater Sensor Networks

There are several challenges [11] faced in the underwater environments which are as follows:

- Propagation and attenuation : Underwater medium mainly uses acoustic signals for communication purpose. These signal have lower speed compared to radio waves. Some of the characteristics of communication in water medium such as scattering, absorption, refraction creates obstacle for signal propagation in underwater medium. Attenuation can be defined as the decrease in the intensity of the signal during propagation. Attenuation is high in underwater medium

- Gathering/collection of data reliably

- Synchronization of the clock time of sensor nodes

- Management of energy in order to secure lifetime of the network[12]

- Localization and security issues with respect to the nodes and their communication in the network

- Available communication link/bandwidth is limited due to the lack of fair channel sharing mechanisms [13].

- Sensor nodes may malfunction or fail due corrosions and other factors of underwater environment.

\section{CHALLENGES}

- The underwater channel is severely impaired, especially due to multi-path and fading;

- Propagation delay in underwater is five orders of magnitude higher than in radio frequency (RF) terrestrial channels, and extremely variable;

- High bit error rates and temporary losses of connectivity (shadow zones) can be experienced, due to the extreme characteristics of the underwater channel;

- Battery power is limited and usually batteries cannot be recharged, also because solar energy cannot be exploited;

- Underwater sensors are prone to failures because of fouling and corrosion. 


\section{PROPOSED SYSTEM}

Mobile sink schemes improves network lifetime. However, previous studies on sink mobility either assume that global information of the network is already available or the mobile sink convey the global information through repeated network wide broadcasting. Thus the gain in network lifetime can be offset by the broadcasting which incurs extra high energy consumption.

Algorithm works as follows :

1. Initially network is initialized by sending activation packet and every node in a network assumes itself as a cluster.

2. Clusters are formed by using distance and residual energy.

3. Cluster Head are chosen from the cluster with highest energy and it changes after each round.

4. $\mathrm{CH}$ aggregates the data send from the cluster node and send it to the sink.

5. After each round sink changed its position randomly so that energy consumption is uniform.

6. If each round $\mathrm{CH}$ has not sufficient energy, then reclustering is done.

\section{CLUSTER HEAD FORMATION}

Among that multiple $\mathrm{CH}$, the present $\mathrm{CH}$ is selected using Sleep Wake pattern. The awake $\mathrm{CH}$ send CHADV message contain Node Id and an Integer Count. The NCH waits for some times ADVWT for receiving all CHADV from all the potential $\mathrm{CH}$. Then the $\mathrm{NCH}$ choose their corresponding $\mathrm{CH}$ which is having minimum Integer Count. If more than a $\mathrm{CH}$ having minimum Integer Count then it chosen the minimum Id. After that the $\mathrm{CH}$ waits for $\mathrm{CH}-\mathrm{JOIN}$ for a period of JOIN-WT.

After $\mathrm{CH}$ selection the data transfer will be done within the cluster. The $\mathrm{NCH}$ send the data to $\mathrm{CH}$ and $\mathrm{CH}$ send it to base station. For intra cluster communication, no need for any control information. For inter communication the route is discovered using the control information.

\section{a) Route Discover}

The route discovering packet is carried by the first data packet from source to destination node due to energy constraints. The source check the existing route for destination, if there is not existing route then the packet will send to destination. After getting the data packet the destination send the acknowledgement along with reverse path to the source [14].

b) Route Maintenance

Each path from source to destination has time property called ROUTE LIFETIME. When the lifetime exceeds the threshold denotes TIMEOUT and inform that the route is invalid. Node uses the same path before threshold the lifetime is reset to " 0 ".

c) Route Retraction

Route lifetime in routing table exceeds the threshold value the route is discarded. If there is data to send the route rediscovery will be done.

\section{d) Periodic Sleeping}

The nodes shut down the transceiver till the next sampling or receiving will be done.

The moving direction (counterclockwise or clockwise) and velocity $\mathrm{v}$ of the sink are both pre-determined.

Therefore the sink only needs to broadcast across the network to inform all sensor nodes of its current location $0 \mathrm{P}$ at the very beginning for just one time.

Later on, as sensor nodes keep record of the original location of the sink, they can reduce the changed angle _ after a time interval $t$ :

$$
\begin{array}{rr}
\mathrm{v}=\theta * \mathrm{R} \\
\Delta \mathrm{t} & \theta=\mathrm{v} * \Delta \mathrm{t} \\
\mathrm{R}
\end{array}
$$

As P0 is known to all, the new location $\mathrm{P} \Delta \mathrm{t}$ can be determined, After the broadcasting finishes, the mobile sink is prepared or collecting data [15]. Here, we assume that the mobile sink stays at a site for a period long enough for the network to complete a round of data collection, and then moves to the next site. 


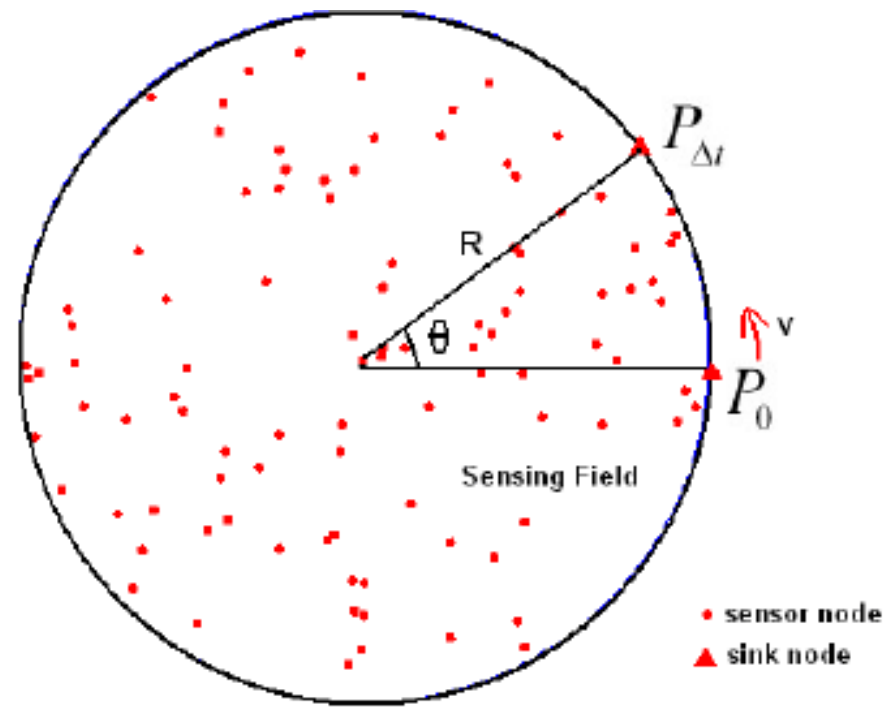

Figure 1 : Sink Relocation

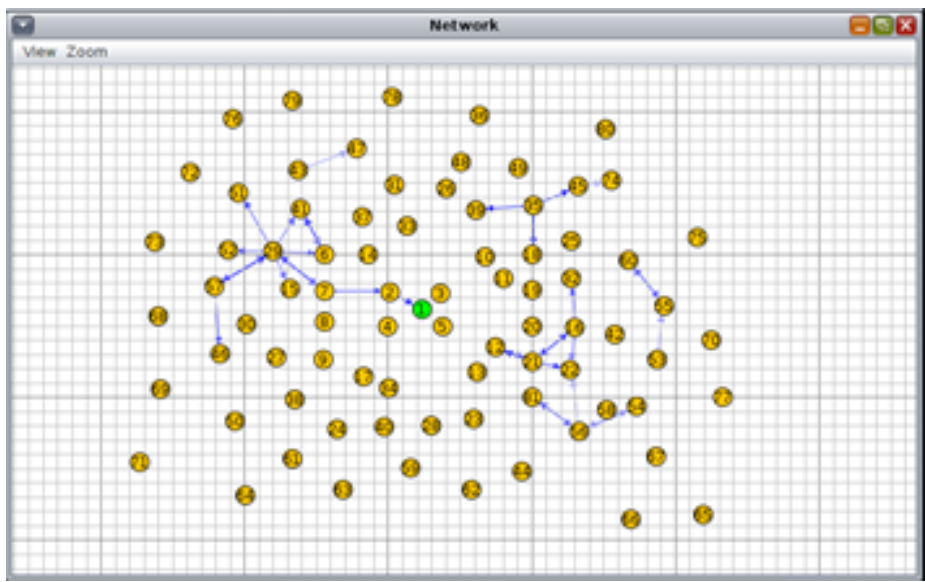

Figure 2 : Cluster Interaction with Mobile Sink
ADVANTAGES:
i) More reliable
ii) Efficient
iii) Consumes less energy
iv) Robust
v) Fault tolerance

\section{IV.CONCLUSION AND FUTURE WORK}

Underwater wireless sensor network (UWSN) uses the acoustic signal which has a unique characteristics like limited bandwidth, high and variable propagation delay, transmit energy, minimum network lifetime and so on. This paper proposes a clustering algorithm adopting a mobile sink and reducing the energy consumption of the nodes does not necessarily reduce the energy dissipation of wireless sensor network. Instead, a careful selection of the nodes and of mobility radius of the sink is required in order to achieve higher energy efficiency compare to a static sink.

Moreover, conclude that in comparison to a static sink placed at the center of the wireless sensor networks, a mobile sink can reduce energy significantly, Irrespective of the mobility radius of the sink. In future, we investigate this approach by using various clustering algorithms. Moreover, we will perform the analysis of the adaptability of our strategy and evaluate its performance.

\section{REFERENCES}

[1] Z. Wei, G. Yang, and Y. Cong, "Security of underwater sensor networks", Chinese Journal of Comuters, vol.35, no.8, pp. 1594-1606, 2012.

[2] Y. Chen, X.M. Xu, and L. Zhang, "Design of RC - LDPC codes and its application for shallow water acoustics communications", Journal of Convergence Information Technology, vol.7, no.12, pp.177-185, 2012. 
[3] F. A. Ian,, P. Dario, and M. Tommaso, "Underwater acoustic sensor networks : Research challenges”, Ad Hoc Networks, vol. 3, no.3, pp. 257279,2005 .

[4] J.H Cui, J. Kong, M. Gerla, and S. Zhou, "Challenges: Building Scalable Mobile Underwater Wireless Sensor Networks for Aquatic Applications", Special Issue of IEEE Network on Wireless Sensor Networking, May 2006.

[5] Liu. L, Zhou. S, and Cui, J.H., "Prospects and Problems of Wireless Communication for Underwater Sensor Networks", WILEY WCMC, Vol.8, Pages $977-994,2008$.

[6] Ying Guo, Yutao Liu, "Time Synchronized for Mobile Underwater Sensor Networks", Journal of Networks, Vol. 8, No.1, January 2013.

[7] Kaminsky, E., "Chirp signaling offers modulation scheme for underwater communications", SPIE Newsroom, 10.1117/2.1200608.0357, (http://newsroom.spie.org/x4310.xml), 3 pp., 2006.

[8] Lee Freitag, Milica Stojanovic, Sandipa Singh, and Mark Johnson, "Analysis of Channel Effects on Direct-Sequence and Frequency-Hopped Spread-Spectrum Acoustic Communication”, IEEE JOURNAL OF OCEANIC ENGINEERING, VOL. 26, NO. 4, OCTOBER 2001

[9] John Heidemann, Milica Stojanovic and Michele Zorzi "Underwater Sensor Networks: Applications, Advances and challenges", Phil. Trans. R. Soc. A (2012), 370, 158 - 175, doi: 10.1098/rsta 2011.0214.

[10] J. Heidemann, W. Ye, J. Wiils, A. Syed, and Y. Li, "Research hallenges and Applications for Underwater Sensor Networking", IEEE Wireless Communications and Networking Conference, Las Vegas, Nevada, USA, April 2006.

[11] Manjula. R and Manvi. S, "Issues in underwater acoustic sensor networks", International Journal of Comuter and Electrical Engineering, 2011.

[12] Sushma V, Shanthi MB, “, Time Synchronized Fair Data Transmission in Secure UWSNs", International Journal of Science and Research (IJSR), ISSN (Online): 2319-7064, Volume 4, Issue 6, June 2015

[13] Vitaly Bokser, Carl Oberg, Gaurav S. Sukhatme and Aristides Requicha, "A Small Submarine Robot For Experiments in Underwater Sensor Networks", department of Computer Science, University of Southern California.

[14] Sushma V, "Clustered Fair Data Transmission in Energy Efficient UWSNs", in International Journal of Advanced Research in Computer and Communication Engineering, ISSN (Online) 2278-1021 ISSN (Print) 23195940 Volume 5, Issue 10, October 2016.

[15] J. Xu, "Energy efficient tree based multi-path power control for underwater sensor networks", IEEE Transaction on Parallel and Distributed Systems, vol.23, pp. 2107-2116,

\section{BIOGRAPHY}

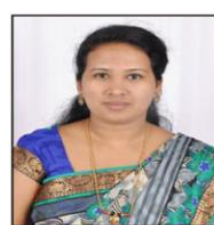

Sushma V has been awarded with B.E and M.Tech degree from Visvesvaraya Technological University. Currently she is working as Assistant Professor in GSSS Institute of Engineering and Technology. Her research interests include optimization in sensor networks, data transmission and security in cloud computing. 